\title{
Quels critères pour l'examen de spécialiste?
}

Ch. Hänggeli, responsable du Secrétariat pour la formation prégraduée, postgraduée et continue (FPPC)

Le Comité central de la FMH (CC) a fixé au $1^{\mathrm{er}}$ janvier 2003 l'introduction du caractère éliminatoire de l'examen de spécialiste pour les spécialités suivantes: allergologie et immunologie clinique, dermatologie et vénéréologie, infectiologie, neurologie, chirurgie orthopédique, médecine pharmaceutique, médecine physique et réadaptation, pneumologie, néonatologie et neuropédiatrie.
Le Comité central a mis en vigueur au $1^{\text {er }}$ janvier 2003 la clause éliminatoire de l'examen de spécialiste pour dix autres spécialités. Ainsi, la réussite de l'examen de spécialiste est désormais exigée pour plus de la moitié des titres, sous réserve des dispositions transitoires applicables.

Nous publions ci-après un aperçu actuel des disciplines où la réussite de l'examen de spécialiste est obligatoire et de celles où seule la participation à l'examen est requise.

\section{Disciplines où la réussite de I'examen de spécialiste est requise pour l'attribution du titre}

Dans les disciplines suivantes, les titres de spécialiste et les attestations de formation approfondie sont attribués uniquement après réussite de l'examen de spécialiste:

\begin{tabular}{lr} 
Titre de spécialiste & Entrée en vigueur \\
\hline Allergologie et immunologie clinique & 1.1 .2003 \\
\hline Anesthésiologie & 2.4 .1986 \\
\hline Cardiologie & 1.1 .1999 \\
\hline Chirurgie (y c. examen de base) & 1.1 .1999 \\
\hline Chirurgie orthopédique & 1.1 .2003 \\
\hline Chirurgie pédiatrique & 2.4 .1986 \\
\hline Dermatologie et vénéréologie & 1.1 .2003 \\
\hline Endocrinologie-diabétologie & 1.1 .2001 \\
\hline
\end{tabular}

\begin{tabular}{|c|c|}
\hline Gastroentérologie & 1.1 .2000 \\
\hline Gynécologie et obstétrique & 1.1.1999 \\
\hline Hématologie & 1.1 .2001 \\
\hline Infectiologie & 1.1 .2003 \\
\hline Médecine du travail & 1.1 .2001 \\
\hline Médecine générale & 1.1 .2000 \\
\hline Médecine intensive & 1.1 .2001 \\
\hline Médecine interne & 1.1 .1999 \\
\hline Médecine légale & 1.1 .2001 \\
\hline Médecine nucléaire & 2.4 .1986 \\
\hline Médecine pharmaceutique & 1.1 .2003 \\
\hline Médecine physique et réadaptation & 1.1 .2003 \\
\hline Médecine tropicale & 1.1 .2001 \\
\hline Neurochirurgie & 2.4 .1986 \\
\hline Neurologie & 1.1 .2003 \\
\hline Oncologie médicale & 1.1 .2001 \\
\hline Ophtalmologie & 1.1 .2002 \\
\hline ORL (sans la chirurgie maxillo-faciale) & 1.1 .2001 \\
\hline Pathologie & 1.1 .1999 \\
\hline Pneumologie & 1.1 .2003 \\
\hline Psychiatrie et psychothérapie & 1.1.2001 \\
\hline $\begin{array}{l}\text { Psychiatrie et psychothérapie d'enfants } \\
\text { et d'adolescents }\end{array}$ & 1.1 .2002 \\
\hline Radiologie & 2.4 .1986 \\
\hline Radio-oncologie / radiothérapie & 2.4 .1986 \\
\hline Rhumatologie & 1.1 .2001 \\
\hline Urologie & 1.1 .2002 \\
\hline Formations approfondies & n vigueur \\
\hline Cardiologie pédiatrique & 1.1 .2001 \\
\hline Néonatologie & 1.1 .2003 \\
\hline Neuropédiatrie & 1.1 .2003 \\
\hline
\end{tabular}




\begin{tabular}{|c|c|c|c|c|c|}
\hline $\begin{array}{l}\text { Tableau } 1 \\
\text { Dois-je réussir l'examen de spécialiste? }\end{array}$ & en vigueur & 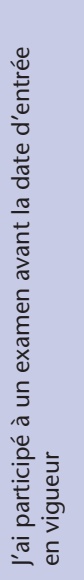 & 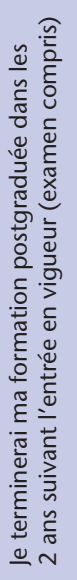 & 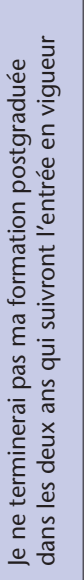 & 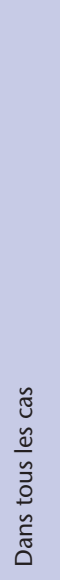 \\
\hline Allergologie et immunologie clinique & 1.1 .2003 & $P$ & $P$ & $\mathrm{R}$ & - \\
\hline Anesthésiologie & 2.4 .1986 & - & - & - & $\mathrm{R}$ \\
\hline Cardiologie pédiatrique & 1.1 .2001 & $P$ & $P$ & $\mathrm{R}$ & - \\
\hline Cardiologie & 1.1 .1999 & $P$ & $P$ & $R$ & - \\
\hline Chirurgie (y c. examen de base) & 1.1.1999 & $\mathrm{P}$ & $\mathrm{P}$ & $\mathrm{R}$ & - \\
\hline Chirurgie orthopédique & 1.1 .2003 & $P$ & $P$ & $\mathrm{R}$ & - \\
\hline Chirurgie pédiatrique & 2.4 .1986 & - & - & - & $\mathrm{R}$ \\
\hline Dermatologie et vénéréologie & 1.1 .2003 & $P$ & $P$ & $\mathrm{R}$ & - \\
\hline Endocrinologie-diabétologie & 1.1 .2001 & $\mathrm{P}$ & $P$ & $\mathrm{R}$ & - \\
\hline Gastroentérologie & 1.1 .2000 & $\mathrm{P}$ & $\mathrm{P}$ & $\mathrm{R}$ & - \\
\hline Gynécologie et obstétrique & 1.1 .1999 & $\mathrm{P}$ & $P$ & $\mathrm{R}$ & - \\
\hline Hématologie & 1.1 .2001 & $P$ & $P$ & $\mathrm{R}$ & - \\
\hline Infectiologie & 1.1 .2003 & $P$ & $P$ & $\mathrm{R}$ & - \\
\hline Médecine du travail & 1.1 .2001 & $P$ & $\mathrm{P}$ & $\mathrm{R}$ & - \\
\hline Médecine générale & 1.1 .2000 & $\mathrm{P}$ & $\mathrm{P}$ & $\mathrm{R}$ & - \\
\hline Médecine intensive & 1.1.2001 & $\mathrm{P}$ & $\mathrm{P}$ & $\mathrm{R}$ & - \\
\hline Médecine interne & 1.1.1999 & $\mathrm{P}$ & $\mathrm{P}$ & $\mathrm{R}$ & - \\
\hline Médecine légale & 1.1 .2001 & $P$ & $P$ & $R$ & - \\
\hline Médecine nucléaire & 2.4 .1986 & - & - & - & $\mathrm{R}$ \\
\hline Médecine pharmaceutique & 1.1 .2003 & $\mathrm{P}$ & $\mathrm{P}$ & $\mathrm{R}$ & - \\
\hline Médecine physique et réadaptation & 1.1 .2003 & $\mathrm{P}$ & $P$ & $\mathrm{R}$ & - \\
\hline Médecine tropicale & 1.1.2001 & $\mathrm{P}$ & $\mathrm{P}$ & $\mathrm{R}$ & - \\
\hline Néonatologie & 1.1 .2003 & $P$ & $P$ & $R$ & - \\
\hline Neurochirurgie & 2.4 .1986 & - & - & - & $\mathrm{R}$ \\
\hline Neurologie & 1.1 .2003 & $P$ & $P$ & $\mathrm{R}$ & - \\
\hline Neuropédiatrie & 1.1 .2003 & $\mathrm{P}$ & $\mathrm{P}$ & $\mathrm{R}$ & - \\
\hline Oncologie médicale & 1.1.2001 & $\mathrm{P}$ & $\mathrm{P}$ & $\mathrm{R}$ & - \\
\hline Ophtalmologie & 1.1 .2002 & $\mathrm{P}$ & $\mathrm{P}$ & $\mathrm{R}$ & - \\
\hline ORL (sans la chirurgie maxillo-faciale) & 1.1 .2001 & $\mathrm{P}$ & $\mathrm{P}$ & $\mathrm{R}$ & - \\
\hline Pathologie & 1.1 .1999 & $P$ & $P$ & $\mathrm{R}$ & - \\
\hline Pneumologie & 1.1 .2003 & $P$ & $P$ & $\mathrm{R}$ & - \\
\hline Psychiatrie et psychothérapie & 1.1 .2001 & $\mathrm{P}$ & $\mathrm{P}$ & $\mathrm{R}$ & - \\
\hline Psychiatrie et psychothérapie d'enfants et d'adolescents & 1.1 .2002 & $\mathrm{P}$ & $\mathrm{P}$ & $\mathrm{R}$ & - \\
\hline Radiologie & 2.4 .1986 & - & - & - & $\mathrm{R}$ \\
\hline Radio-oncologie / radiothérapie & 2.4 .1986 & - & - & - & $\mathrm{R}$ \\
\hline Rhumatologie & 1.1 .2001 & $P$ & $P$ & $\mathrm{R}$ & - \\
\hline Urologie & 1.1 .2002 & $P$ & $\mathrm{P}$ & $\mathrm{R}$ & - \\
\hline tous les autres domaines & & - & - & - & $\mathrm{P}$ \\
\hline \multicolumn{6}{|c|}{$\begin{array}{l}\text { Réussite = R; Participation = P. } \\
\text { La documentation relative à la formation postgraduée (avec les dates actuelles d'examen) peut être } \\
\text { consultée sur le site internet de la FMH à l'adresse suivante: www.fmh.ch/awf. Les dates d'examen } \\
\text { seront aussi publiées dans le Bulletin des médecins suisses (BMS). Pour de plus amples informations, } \\
\text { veuillez vous adresser au Secrétariat FPPC de la FMH, case postale 293, Elfenstrasse 18, } 3000 \text { Berne 16, } \\
\text { tél. } 03135911 \text { 11, fax } 03135911 \text { 12, e-mail: diplome@hin.ch. }\end{array}$} \\
\hline
\end{tabular}

\section{Dispositions transitoires}

Font exception les candidats remplissant une des deux conditions suivantes:

- Les candidats ayant déjà participé à un examen de spécialiste (entièrement ou en partie) avant l'entrée en vigueur du caractère éliminatoire de l'examen ne devront pas passer d'autre examen.

- Les candidats terminant leur formation postgraduée dans les deux ans après l'entrée en vigueur du caractère éliminatoire de l'examen de spécialiste ne doivent attester, pour l'obtention du titre, que leur participation à l'examen. Attention: toutes les conditions doivent être remplies durant les deux ans qui suivent la date d'entrée en vigueur (y compris l'examen de spécialiste complet, sauf si la société de discipline médicale refuse la participation à la $2^{\mathrm{e}}$ partie de l'examen en raison d'un résultat insuffisant à la $1^{\text {re }}$ partie)!

\section{Disciplines où seule l'attestation de participation à l'examen de spécialiste est exigée pour l'attribution du titre}

Dans toutes les autres disciplines, une attestation de participation à l'examen doit être présentée pour l'obtention du titre de spécialiste ou d'une formation approfondie. La réussite de l'examen

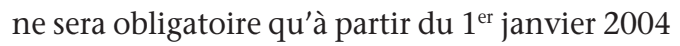
au plus tôt. Cette disposition s'applique également aux disciplines susmentionnées qui bénéficient d'un délai transitoire de deux ans. 